\title{
Mechanism of Removing Monodisperse Gold Particles from a Suspension Using Cuprammonium Regenerated Cellulose Hollow Fiber (BMM Hollow Fiber)
}

\author{
Takashi Tsurumi, Naoki Osawa, Tomoko Hirasaki, \\ Kazuhito Yamaguchi, ${ }^{*}$ Sei-ichi Manabe, \\ and Takashi YAMASHIKI
}

\author{
BMM Project, Fibers and Textiles Administration, Asahi Chemical Industry Co., Ltd., \\ The Imperial Tower 18F, 1-1 Uchisaiwaicho 1-chome, Chiyoda-ku, Tokyo 100, Japan \\ * Institute of Laboratory Animals, Yamaguchi University \\ School of Medicine, Ube, Yamaguchi 755, Japan
}

(Received September 22, 1989)

\begin{abstract}
In order to clarify the mechanism of removing viruses with cuprammonium regenerated cellulose hollow fiber (BMM hollow fiber), monodisperse gold particles were used. The dependences of the concentration of gold particles in the filtrate on the particle concentration and the particle size were investigated. The particles were considered to be caught by BMM through two mechanisms, that is, plugging of capillaries and trapping within voids. Here, the capillaries stand for the narrow pathway among neighboring cellulose particles which construct the membrane and the voids the bulky space surrounded by the aggregated cellulose particles. In the initial stage, the plugging of the capillaries causes decrease in the particle concentration. On the other hand, trapping leads to the occupation of spaces within the voids. When the voids near the inner surface are occupied by gold particles, the particles proceed inwards through channels formed by voids and finally flow out from the outer surface of the membrane. This leads to increase in the particle concentration in the preceding stage. The removability of the particles depends both on the relative size bewtween the capillary (or the void) and the particle and on the trapping capacity.

KEY WORDS Regenerated Cellulose / Hollow Fiber / Gold Particle / Virus / Membrane Structure / Ultrafiltration /
\end{abstract}

In the 19th century, viruses were named a filtrable microbe because of their filtrability through a porcelain plate. In 1937, Elford used a membrane filter prepared from collodion solution in order to decide the size of viruses. ${ }^{1}$ From this result, a virus was known to be a particle larger size than protein molecule. He imaged the membrane was constructed with the straight through cylindrical pores and viruses were caught on the membrane surface not in its inside. Recently, this sieving mechanism of viruses with the membrane was again applied to decide the dimension of nonAnonB hepatitis virus. $^{2}$ That is, the straight through cylindrical pores in the membrane were coated with albumin so as to minimize the adsorption of virus by the polymeric membrane. He assumed that the pore size distribution was very sharp such as being expressed as $\delta$ function and that all viruses having larger diameter than pore size were caught only on the membrane surface. When an appropriate membrane having smaller pore size than the size of virus is used in a laboratory the filtrate obtained may become virus-free, but this membrane is not adequate for industrial usage. This is because it cannot meet the following demands to the filtration performance:

1) The virus logarithmic rejection coefficient $\Phi_{\mathrm{v}}$ defined by eq 1 is not less than 4 , 


$$
\Phi_{\mathrm{v}}=\log C_{\mathrm{o}} / C_{\mathrm{f}}
$$

where $C_{\mathrm{o}}$ and $C_{\mathrm{f}}$ are virus concentrations of the original solution and filtrate, respectively.

2) The sieving coefficient (SC) of proteins is larger than $90 \%$.

3) The filtration capacity, that is, the filtration volume in which the virus removability and the protein permeability remain above the levels shown in above items of 1) and 2), is more than $301 \mathrm{~m}^{-2}$.

The regenerated cellulose hollow fiber (BMM) developed by us could satisfy the above four demands in the cases of human immunodeficiency virus (HIV-1) ${ }^{3}$ and hepatitis B virus (HBV). ${ }^{4}$ However it has not yet been clarified what kinds of mechanisms contribute dominantly to catch viruses with this membrane.

In this article we show that the geometric or steric effect is sufficient to remove viruses by this membrane. That is, the filtration characteristics of BMM that the particles are easily and nearly completely removable from the aqueous solution containing proteins using BMM will be explained from the steric effect. In order to evaluate the contribution of this effect without other effects such as adsorption, we use the colloidal solution of gold particles. These particles do not aggregate and are stable toward mechanical stress such as filtration and particle size distribution is narrow and also adsorption to the membrane is negligible. Then, gold particle whose size is designed so as to be equivalent to the virus in question is a most ideal model substance of the virus to investigate the filtration properties of BMM.

\section{EXPERIMENTAL}

\section{Sample Preparation}

(a) BMM: The hollow fibers were prepared from cellulose cuprammonium solution by the microphase separation method under strict control of the spinning condition. ${ }^{5,6}$ The hollow fibers were bundled into M-module having effective filtration area of $0.03 \mathrm{~m}^{2}$.

(b) Monodisperse gold particles: The particles of given size were prepared by the reduction method of the gold compound of $\mathrm{HAuCl}_{4}$. Although, the principle of the preparation is given in the literature, ${ }^{6}$ we used a minor improved method by the addition of polyethylene glycol with average molecular weight of 20000 in order to make stable dispersed solution. The particle size distribution was evaluated using transmission electron microscope.

\section{Measurement}

(a) Pore characteristics: The mean pore size $2 \bar{r}_{\mathrm{f}}$ was calculated using eq 2 by putting observed values of the filtration rate of water $J$ $(\mathrm{ml} \mathrm{min}-1)$, the porosity determined by the apparent density of BMM $P_{\mathrm{r}} \rho$, the transmembrane pressure $\Delta P(\mathrm{mmHg})$ and the viscosity of water $\eta$ (centipoise),

$$
2 \bar{r}_{\mathrm{f}}=2.0 \times\left(J \cdot d \cdot \eta / \Delta P \cdot A \cdot P_{\mathrm{r}} \rho\right)^{1 / 2}
$$

(b) Ultrafiltration: The filtration was carried out at $20^{\circ} \mathrm{C}$ under a constant pressure of $100 \mathrm{mmHg}$. The dependence of the concentration of gold particles in the filtrate on filtration time, volume and the concentration of the gold particles in original solution was investigated.

(c) Electron microscopy: The frequency distribution of the gold particles present in BMM was observed through transmission electron microscopy of an ultrathin cross section of BMM after filtration. The section with thickness of 60 to $80 \mathrm{~nm}$ was prepared through ultramicrotome ("Ultratome III" manufactured by LKB in Sweden) using a diamond knife ("Diatome").

(d) Particle size and its zeta potential: The size of particles was observed by electron microscopy and the zeta potential of this particle was evaluated from its electropheresis behavior using ELS-800 manufactured by Otsukadenshi Co., Ltd., Japan.

(e) Concentration of gold particles: The concentration of gold particles was measured 
using spectrophotometry at $530 \mathrm{~nm}$ of wave length.

\section{RESULTS}

Table I summarizes some characteristic values of the colloidal solution of gold. Here, the $i$-th mean particle size $d_{\mathrm{g} i}$ is defined as

$$
d_{\mathrm{g} i}=\frac{\int_{0}^{\infty} d_{\mathrm{g}}^{i} N\left(d_{\mathrm{g}}\right) \mathrm{d} d_{\mathrm{g}}}{\int_{0}^{\infty} d_{\mathrm{g}}^{i-1} N\left(d_{\mathrm{g}}\right) \mathrm{d} d_{\mathrm{g}}} \quad(i=1,2, \text { and } 3)
$$

where $d_{\mathrm{g}}$ is the diameter of the gold particles and $N\left(d_{\mathrm{g}}\right)$ is the frequency distribution function defined as the existing probability of particles whose size is between $d_{\mathrm{g}}$ and $d_{\mathrm{g}}+\mathrm{d} d_{\mathrm{g}}$ is expressed by $N\left(d_{\mathrm{g}}\right) \mathrm{d} d_{\mathrm{g}}$. The ratio between $d_{\mathrm{g} 3}$ and $d_{\mathrm{g} 2}$ reflects the width of the distribution. Judging from the experimental value of $d_{\mathrm{g} 3} / d_{\mathrm{g} 2}$, all samples of G20, G30, G50, and G60 can be regarded as having monodispersed size distribution. The zeta potential of G30 was $-25 \mathrm{mV}$ and other particles may have negative charge due to the same preparation procedure for G30. The membrane surface of BMM in

Table I. Characteristics of a colloidal solution of gold

\begin{tabular}{|c|c|c|c|c|}
\hline Sample code & G20 & G30 & G50 & G60 \\
\hline \multicolumn{5}{|l|}{$\begin{array}{l}\text { Average particle } \\
\text { diameter } / \mathrm{nm}\end{array}$} \\
\hline$d_{\mathrm{g} 1}$ & 18.3 & 30.0 & 49.4 & 60.7 \\
\hline$d_{\mathrm{g} 2}$ & 21.1 & 35.4 & 53.6 & 62.2 \\
\hline$d_{\mathrm{g} 3}$ & 22.7 & 38.2 & 59.1 & 63.7 \\
\hline$d_{\mathrm{g} 3} / d_{\mathrm{g} 2}$ & 1.07 & 1.08 & 1.10 & 1.02 \\
\hline Zeta poential/mV & $-^{a}$ & -25 & $-^{a}$ & $-^{\mathrm{a}}$ \\
\hline $\begin{array}{l}\text { Particle conc. of } \\
\text { original solution } \\
\text { /ppm }\end{array}$ & 58 & 58 & 58 & 58 \\
\hline $\begin{array}{l}\text { Number of particles } \\
\qquad / \times 10^{11} \cdot \mathrm{ml}^{-1}\end{array}$ & 9.36 & 2.12 & 0.48 & 0.26 \\
\hline
\end{tabular}

a Not tested. water of $\mathrm{pH}=7.0$ has slightly negative charge and then the electrostatic repulsion force may work between the particles and membrane surface. The degree of the adsorption of gold particles onto $\mathrm{BMM}$ in water at $20^{\circ} \mathrm{C}$ was below the detectable level as expected from

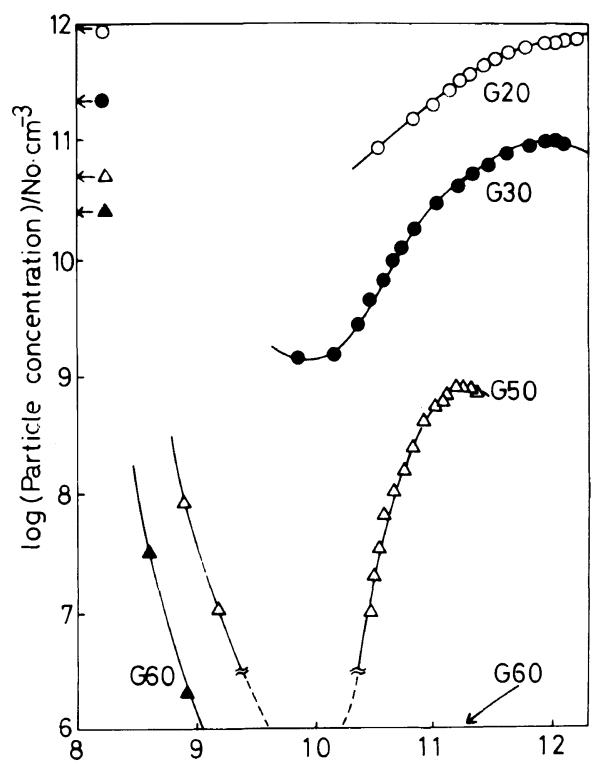

$\log$ (Total number of challenge particles) $/ \mathrm{No} \cdot \mathrm{cm}^{-2}$

Figure 1. Change in filtrate concentration of gold particles with total challenge number of particles for gold particle with various sizes. The arrows at the left hand side indicate the concentrations of the original solutions.

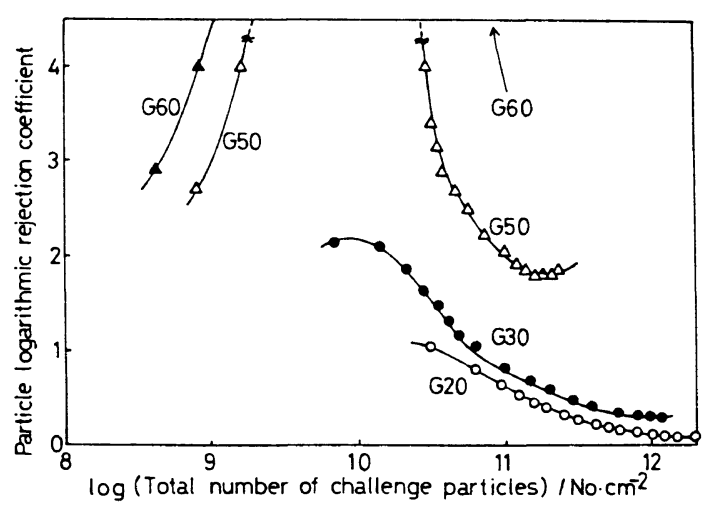

Figure 2. Change in particle logarithmic rejection coefficient with total challenge number of particles for gold particle with various sizes. The arrow attached at G60 indicates the value is more than 4 . 
their electrostatic repulsion force.

Figure 1 shows the change in the gold particle concentration of the filtrate with increased total challenge number of the particles $V$. Figure 2 shows the change in particle logarithmic reduction coefficient $\Phi_{\mathrm{g}}$ from the data in Figure 1 . The $\Phi_{\mathrm{g}}$ value increased in a very small range of $V$ such as less than $10^{10} \mathrm{No} \mathrm{cm}^{-2}$ and took a maximum value and decreased gradually. When the value of $V$ exceeded $10^{11} \mathrm{No} \mathrm{cm}^{-2}$, $\Phi_{\mathrm{g}}$ approached an asymptotic values of 0.05 and 0.3 in the cases of two samples of G20 and G30, respectively, and showed minimum value of 1.8 for G50 and kept high level of more than 4 for G60. The $\Phi_{\mathrm{g}}$ value was always larger for the larger particle size when the comparison was made under the same challenge number, indicating that the size of a particle governs its removability for BMM as expected. It may be deduced from the complicated behavior in the curve of $\Phi_{\mathrm{g}} v s . V$ shown in Figure 2 that there are, at least, two different ways for gold particles in BMM to affect $\Phi_{\mathrm{g}}$ value; that is, giving rise to increase in $\Phi_{\mathrm{g}}$ and decrease in $\Phi_{\mathrm{g}}$. These two ways will be discussed in more detail in the latter section.

Figure 3 shows the curves of $\Phi_{\mathrm{g}} v s$. $V$ of G30 with various concentrations of G30 in the original solution. The particle concentration of the filtrate decreased with decreasing concentration of the original solution. The $\Phi_{\mathrm{g}}$ value increased with decrease in the concentration of the original solution ( $c f$. Figure 4). When we compare the removability of particles with different concentrations under the same challenge number of particles, BMM can catch more particles easily from the poorer particle solution. The influence of the particle concentration on $\Phi_{\mathrm{g}}$ appears stronger in the region of $V$ higher than $10^{10} \mathrm{No} \mathrm{cm}^{-2}$.

Figure 5 shows transmission electron micrographs of cross sections of BMM after the filtration of G30. With increase in the total challenge number of particles the density of population increases and the area where a particle cannot be found expands into the inside

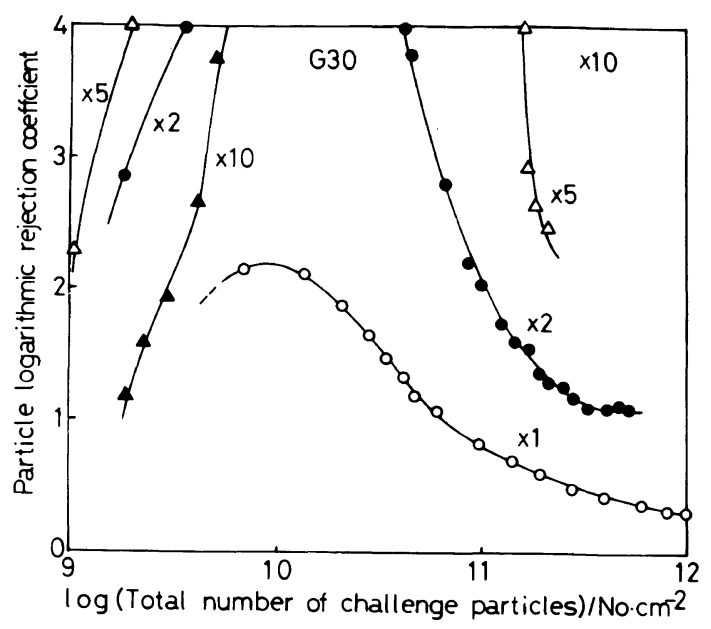

Figure 3. Dependence of particle logarithmic rejection coefficient of G30 on total challenge number of gold particles for the solution with various particle concentrations. The figures indicate dilution times.

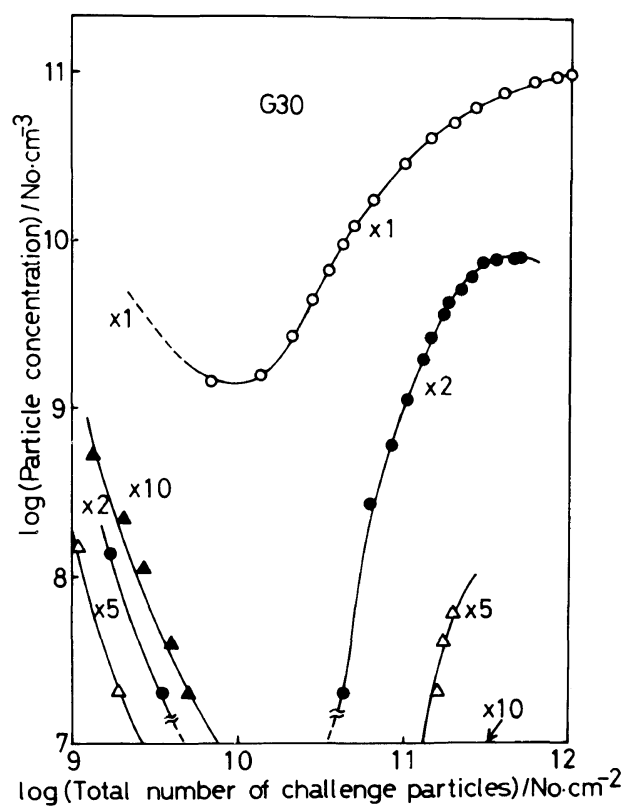

Figure 4. Dependence of filtrate concentration of gold particles of G30 on total challenge number of gold particles for the solution with various particle concentrations. The figures indicate dilution times.

of the wall of BMM. By counting the number of particles in a given area of BMM, we get the distribution of gold particles in BMM. 
T. TSurumi et al.

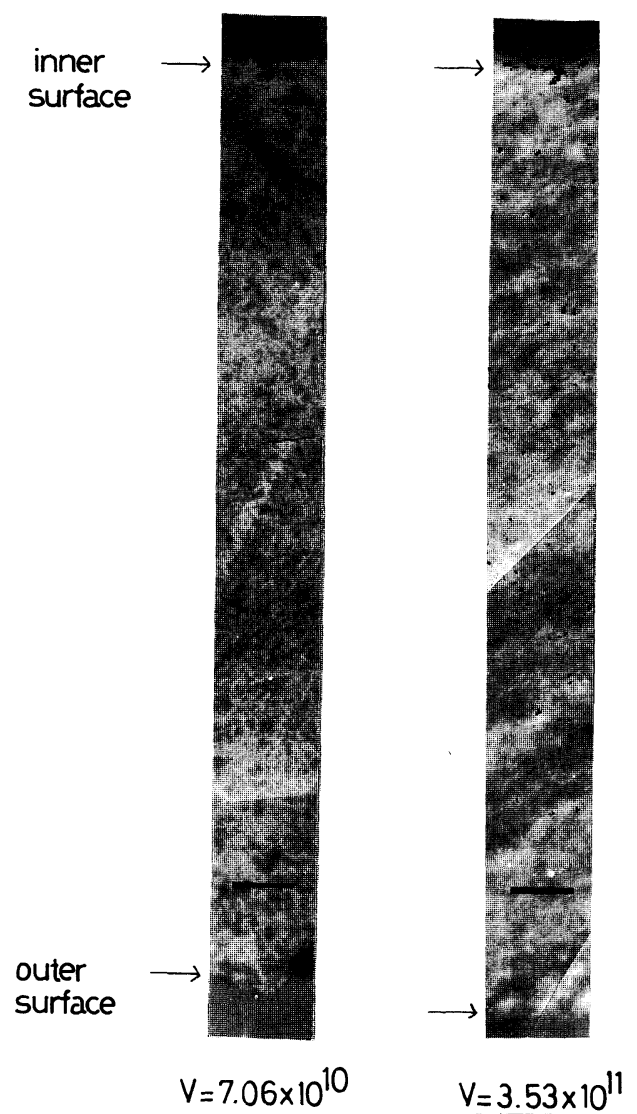

Figure 5. Transmission electron micrograph of BMM after filtration of G30.

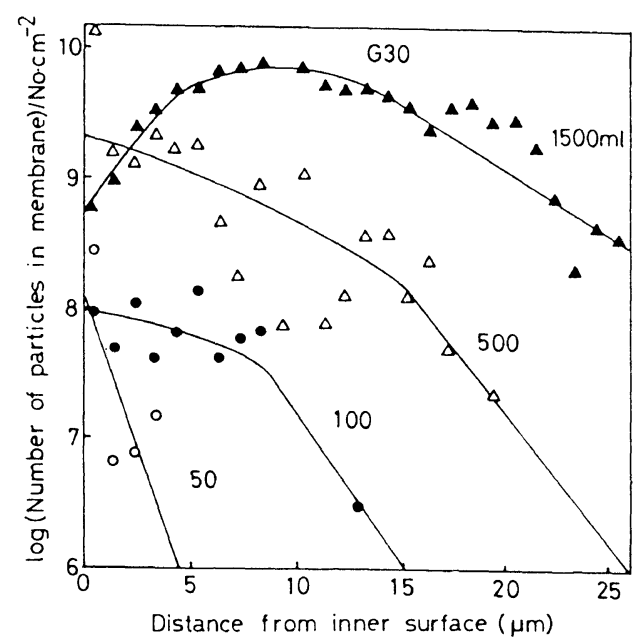

Figure 6. Dependence of total number of gold particles of $\mathrm{G} 30$ in unit area on distance from the inner surface. The figures indicate filtration volume.

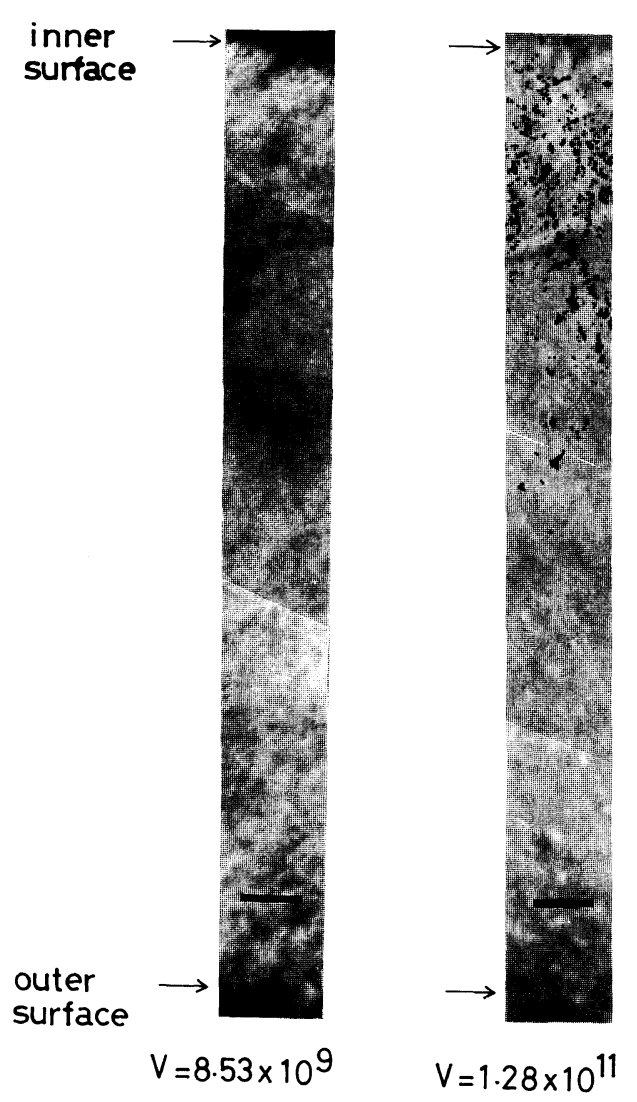

Figure 7. Transmission electron micrograph of BMM after filtration of G60.

Figure 6 gives the number of particles in a unit area of a cross section of BMM $\mathrm{d} N / \mathrm{d} x$ as a function of the distance $x$ from the inner surface. When the challenge number increases the curve of $\mathrm{d} N / \mathrm{d} x$ vs. $x$ shifts to the upper side. This indicates that the total number of the particles caught in BMM increases and the particles move to the inside of BMM wall with increase of the challenge number. The slopes of these curves in the region of $x$ larger than the value where $d N / d x$ shows a maximum value can be represented by a straight line.

Figures 7 and 8 show the transmission electron micrographs of the cross section of BMM after filtration and the curves of $\mathrm{d} N / \mathrm{d} x$ vs. $x$ for the case of G60, respectively. The changes in Figures 7 and 8 are similar to the 


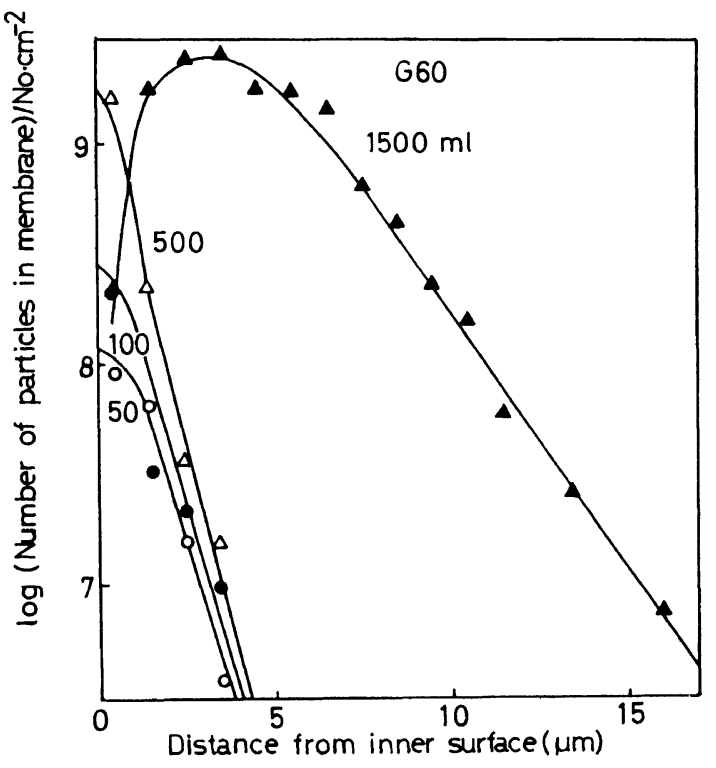

Figure 8. Dependence of the total number of gold particles of G60 in unit area on distance from the inner surface. The figures indicate filtration volume.

Table II. Capture coefficients of G30 and G60 for various filtration volumes

\begin{tabular}{ccc}
\hline & \multicolumn{2}{c}{$\mu_{\mathrm{g}} / \mu \mathrm{m}^{-1}$} \\
\cline { 2 - 3 } Filtration volume $/ \mathrm{ml}$ & $\mathrm{G} 30$ & $\mathrm{G} 60$ \\
\hline 50 & 0.71 & 1.06 \\
100 & 0.61 & 1.45 \\
500 & 0.68 & 1.46 \\
1500 & 0.66 & 0.81
\end{tabular}

cases of Figures 5 and 6, respectively. Then, we can generally describe the dependence of $\mathrm{d} N / \mathrm{d} x$ on $x$ as following an approximation equation in the decline region of $\mathrm{d} N / \mathrm{d} x$,

$$
\mathrm{d} N / \mathrm{d} x=N_{\mathrm{p}_{0}} \exp \left(-\mu_{\mathrm{g}} x\right)
$$

where $\mu_{\mathrm{g}}$ is defined as the capture coefficient and is independent of $x$ but depends on particle size, the total challenge numbers and pore characteristics, and $N_{\mathrm{p}_{0}}$ is the extrapolated value of $\mathrm{d} N / \mathrm{d} x$ at $x=0$ and changes depending on the filtration conditions and pore characteristics. Table II summarizes the value of $\mu_{\mathrm{g}}$ obtained for various filtration volumes for $\mathrm{G} 30$ and G60. When the conditions are same between G30 and G60, the value of $\mu_{\mathrm{g}}$ for G60 is always larger than that for G30. Then, particle size is the main factor in deciding the particle removability of BMM.

\section{DISCUSSION}

Figure 1 and Table II indicate that BMM has the ability to remove gold particles by its geometric pore structure.

In order to discuss the mechanism of removing gold particles with BMM, it is indispensable to analyze thoroughly the pore structure of the membrane and we have performed already the analysis as to BMM with mean pore size of $30 \mathrm{~nm} .^{7}$ According to this analysis this membrane has cellulose particles having an average diameter of about $40 \mathrm{~nm}$ (referred to as secondary particles) as its structure unit. Therefore the membrane has two types of pores, one of which is the pore formed among neighboring secondary particles (referred to as capillaries) and the other is that formed as a vacant space among aggregates of the secondary particles (referred to as voids).

Figure 9 shows a schematic representation of the pore structure of the membrane. ${ }^{8}$ Capillaries are very narrow and constitute a large number of channels (A) linking themselves throughout the whole thickness of the membrane. The pores formed by voids are large and classified into three types: the first is the pore which is linked to other voids only by capillaries (B), the second is the pore which is neighbored by several voids and open to either inner surface or outer surface (C) (not depicted in the figure) and the third is the pore which constitute channels penetrating the membrane from one surface to the other surface (D).

When the gold particles are filtered with BMM with capillaries whose diameter is much larger than that of the gold particle, they never plug capillaries but may stay in the space within the void where the velocity of streamline of 

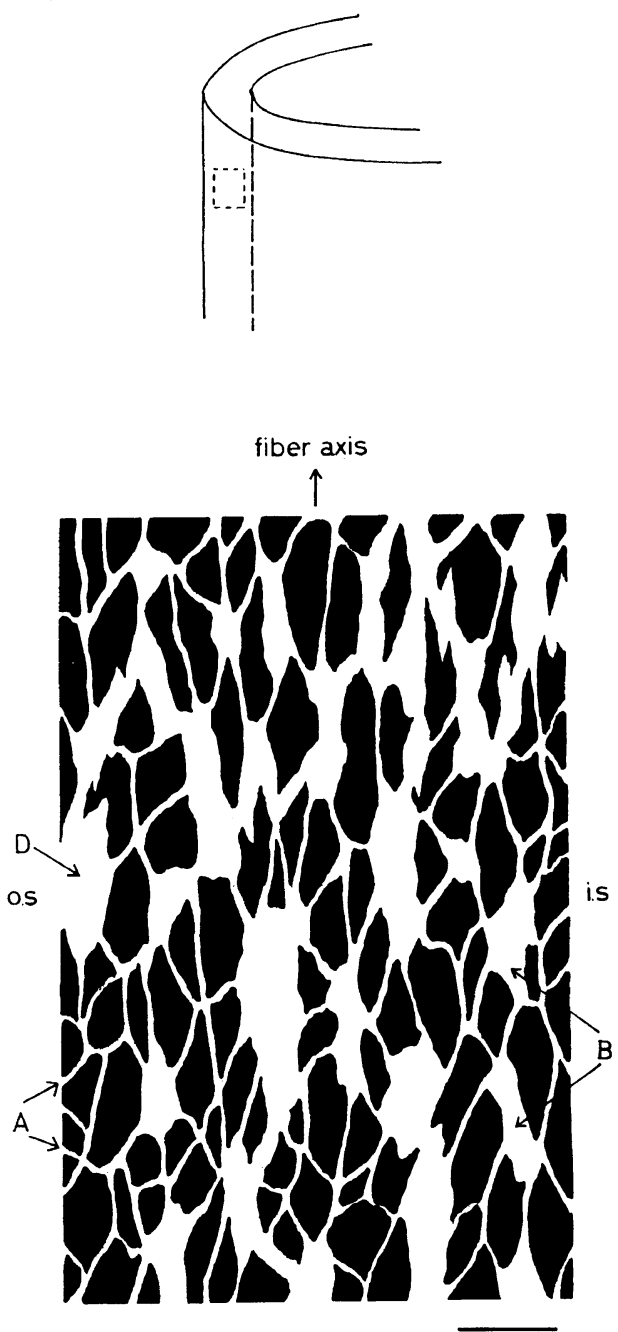

Figure 9. Schematic representation of pore structure of a cross sectional view perpendicular to the fiber axis for BMM 30. Black blocks stand for aggregates of secondary particles of cellulose and white zone stands for pore channel. Scale stands for $500 \mathrm{~nm}$.

liquid is very low or nearly zero. We should like to call this mechanism 'trapping' hereafter. When the trapping spaces near the inner surface are occupied by the gold particles, the particles proceed inwards through capillaries or voids and there occupy other trapping spaces. In this way gold particles proceed toward the outer surface gradually and flow out from the membrane. As this process proceeds the concentration of the gold particles in filtrates increases. When all trapping spaces available are consumed, the concentration of gold particles in filtrate reaches its maximum level. This is the case of G20. The slight decline of concentration at the end of filtration may be due to the plugging of capillaries.

When the diameter of gold particles is a little smaller than or nearly equals the diameter of the capillaries of the membrane the plugging of the capillaries occurs in the early stage. This plugging give rise to decrease in the concentration of gold particles in filtrates ( $c f$. Figures 1 and 4) and increase in the particle logarithmic rejection coefficient at the early stage of filtration ( $c f$. Figures 2 and 3). Along with this, gold particles are trapped in voids in the same way as the case of G20. But when the gold particles occupy the trapping space near the inner surface, they proceed inwards more frequently through neighboring voids than through capillaries because the latter is not large enough to pass through in this case. The larger the size of gold particles becomes, the more difficult their progress towards the outer surface becomes. This causes decrease of the filtrate concentration with larger gold particles. This is the case of G30 and G50.

When the diameter of gold particles is larger than that of capillaries, gold particles cannot pass through the capillaries and are plugged in them. Gold particles move only through channels formed by voids. If there are no channels formed by voids which penetrate the membrane, gold particles cannot pass through the membrane and the filtrate concentration remains below the detectable level. This is the case of G60. However, judging from the electron micrograph where gold particles are observed to proceed to the outer surface, possibly there are some channels formed by voids which penetrate the membrane. In the case of gold particles with the diameter larger than that of the channels, most of them will accumulate on the inner surface, not entering the inside of the membrane. 
Summing up the above discussion it can be concluded that gold particles are caught by BMM through two mechanisms, one is plugging of capillaries and the other trapping within voids. In the early stage of filtration gold particles are plugged into capillaries which leads to decrease in the filtrate concentration. On the other hand trapping within voids starts from the beginning of filtration and gold particles gradually accumulate within voids and consume trapping spaces successively. Gold particles then proceed towards the outer surface through channels formed by voids and flow out from the membrane. The latter mechanism resembles that of adsorption column, where solutes flow out from the column after there is no more adsorption capacity.

\section{REFERENCES}

1. W. J. Elford, Proc. R. Soc. London, Ser. B, 112, 384 (1933).

2. L. He, D, Alling, T. Popkin, M. Shapiro, H. J. Alter, and R. H. Purcell, J. Infectious Diseases, 156, 636 (1987).

3. Y. Hamamoto, S. Harada, S. Kobayashi, K. Yamaguchi, H. Iijima, S. Manabe, T. Tsurumi, H. Aizawa, and N. Yamamoto, Vox Sang., 56, 230 (1989).

4. S. Sekiguchi, K. Ito, M. Kobayashi, H. Ikeda, T. Tsurumi, G. Ishikawa, S. Manabe, M. Satani, and T. Yamashiki, Membrane, 14, 101 (1989).

5. S. Manabe, M. Iwata, and M. Inoue, Japanese Patent Application, 58-89625 (1983)

6. S. Iwata, S. Manabe, and M. Inoue, Japanese Patent Application, 59-204911 (1984).

7. G. Frens, Nature Phys. Sci., 241, 20 (1973).

8. T. Tsurumi, N. Osawa, H. Hidaka, S. Manabe, K. Yamaguchi, and T. Yamashiki, Polym. J., to be submitted. 